\title{
Far-infrared array reciever (FAR) for SOFIA
}

\section{Christopher K. Walker, Christopher E. Groppi, Christian Y. Drouet d'Aubigny, Gondon Chen, Rudolf T. Schieder, et al.}

Christopher K. Walker, Christopher E. Groppi, Christian Y. Drouet d'Aubigny, Gondon Chen, Rudolf T. Schieder, Gopal Narayanan, Arthur W. Lichtenberger, Peter H. Siegel, "Far-infrared array reciever (FAR) for SOFIA," Proc. SPIE 4014, Airborne Telescope Systems, (20 June 2000); doi: 10.1117/12.389093 


\title{
A Far-infrared Array Receiver (FAR) for SOFIA
}

\author{
C.K. Walker ${ }^{a}$, C.E. Groppi ${ }^{a}$, C.Y. Drouet d'Aubigny ${ }^{a}$

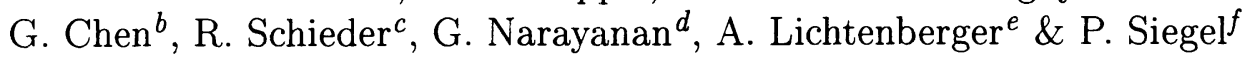 \\ ${ }^{a}$ Steward Observatory, The University of Arizona \\ 933 N Cherry Ave. Tucson, AZ 85721 USA \\ ${ }^{b}$ Goddard Spaceflight Center, Laboratory of Extraterrestrial Physics, Greenbelt, MD 20771 USA \\ ${ }^{c}$ Univ. Koeln, I. Physik Inst. Zuelpicher Strasse 77, Koeln, D-500 41 Germany \\ ${ }^{d}$ University of Massachusetts, FCRAO, Lederle GRC, Amherst, MA 01003 USA \\ ${ }^{e}$ Dept. of Electrical Engineering, Univ. of Virginia, Charlottesville, VA 22903 USA \\ ${ }^{f}$ California Institute of Technology, JPL, Pasedena, CA 91009 USA
}

\begin{abstract}
In this paper, we present the design for a 16-channel heterodyne array receiver for use on SOFIA. The array will be capable of using either hot-electron bolometers or membrane mounted Schottky diodes in efficient, low-cost waveguide mounts. Focal plane arrays will be constructed to target astrophysically important lines between $~ 1.9$ and $3 \mathrm{THz}$. Due to the prevailing physical conditions in the interstellar medium, this frequency range is one of the richest in the FIR portion of the spectrum. An array receiver designed for this wavelength range will make excellent use of the telescope and the available atmospheric transmission, and will provide a new perspective on stellar, chemical, and galaxy evolution in the present as well as past epochs. The proposed system uses the most sensitive detectors available in an efficient optical system. The local oscillator will be a compact $\mathrm{CO}_{2}$ pumped far-infrared laser specifically developed for SOFIA. The backend spectrometer will be a $4 \times 4$ array of acousto-optic spectrometers (AOS). The spectrometer utilizes proven hardware and technologies to provide broadband performance $(\geq 1 \mathrm{GHz}$ per AOS channel) and high spectral resolution (1 MHz). The proposed instrument will be the fastest and most sensitive heterodyne receiver ever to operate in its wavelength regime.
\end{abstract}

Keywords: Far-Infrared, Array Receiver, SOFIA

\section{INTRODUCTION}

A few of the most important molecular and atomic species which the instrument will sample are $\mathrm{CII}, \mathrm{OI}, \mathrm{CO}, \mathrm{OH}$, $\mathrm{NII}, \mathrm{CH}, \mathrm{H}_{2}^{18} \mathrm{O}$, and $\mathrm{HD}$. These are important as diagnostics of a wide variety of astronomical phenomena including interstellar clouds, star-forming regions, young stellar objects, circumstellar envelopes, planetary nebulae, starburst galaxies, and planetary atmospheres. The high spatial and spectral resolution of the array will be crucial in many applications, e.g., the study of Galactic star-forming regions, where structure is expected on spatial scales of less than 10 arcseconds and velocity scales of a few $\mathrm{km} \mathrm{s}^{-1}$. The number and types of possible observational projects for the proposed instrument are virtually limitless.

The high sensitivity, spatial coverage, and spectral resolution of the proposed heterodyne array will for the first time permit large numbers of high-quality, high-resolution spectra of many low-excitation transitions of abundant atomic and molecular species to be obtained. Our proposed instrument will be $\sim 20$ times more sensitive per pixel and have 16 times the spatial coverage of earlier heterodyne instruments flown on the KAO.

The potential power of FAR in probing conditions in and around molecular clouds is illustrated in Figure 1. In the middle of the figure we show an image of NGC 6334 made in CO J=4 $\rightarrow 3$ from AST/RO with a receiver system constructed in the PI's lab. The small square of emission in the top right hand corner is the size of the region that could be mapped in CII at full beam spacings with a single receiver channel in one flight ( $\sim \mathrm{hrs})$. The second

Further author information: (Send correspondence to C.K.W.)

C.K.W.: E-mail: cwalker@as.arizona.edu 


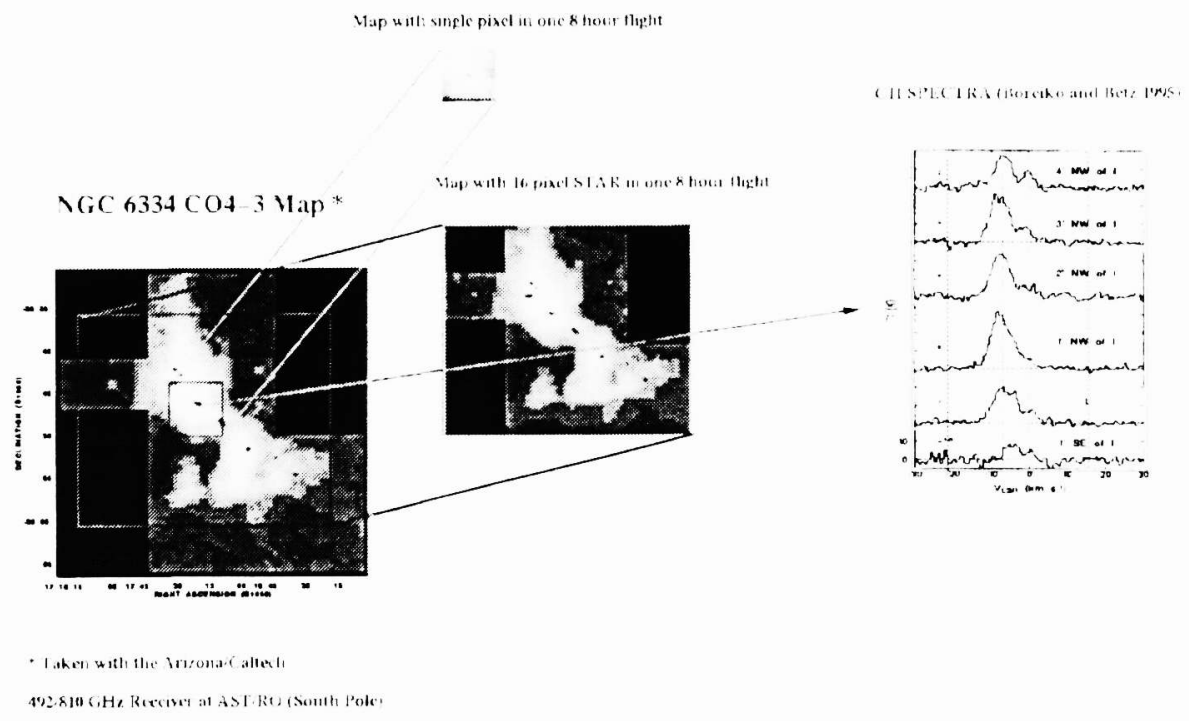

Figure 1. Imaging Capabilities of FAR. Simulated performance advantage of FAR over a single pixel receiver in mapping large scale molecular cloud cores. Each pixel of FAR was assumed to have the same receiver noise temperature as the single pixel receiver. We assumed a conservative receiver noise temperature of $1000 \mathrm{~K}$, and an atmospheric opacity of 0.125 . The SNR in the calculations was 10.

square shows the region that could be mapped with FAR in the same period of time, essentially the whole cloud. The CII spectra at the bottom left were taken by Borieko and Betz $(1995)^{1}$. The width and complexity of the line profiles are well matched to the $150 \mathrm{~km} / \mathrm{s}$ velocity coverage and $0.125 \mathrm{~km} / \mathrm{s}$ velocity resolution available in each FAR pixel.

\section{INSTRUMENT DESCRIPTION}

\subsection{Design Philosophy}

The design goals of the proposed instrument are to achieve the maximum sensitivity, sky-coverage, bandwidth, and tunability with the minimum of complexity and cost. To achieve these goals, we will utilize four new break-through technologies;

1. hot-electron bolometers (HEBs) and monolithic membrane diodes (MOMEDs)

2. laser micromachined waveguide components

3. a reliable, compact, high-power, submillimeter laser system

4. an efficient, broadband, high resolution, array AOS

We will first provide a brief description of the proposed instrument and then describe how we will apply these new technologies to its construction.

\subsection{Block Diagram}

A block diagram of FAR is shown in Figure 2. After emerging from the instrument flange the beam first passes through an LO diplexer which efficiently injects the local oscillator beams into the signal path. The diplexer consists of a single, $3.162 \mathrm{~mm}$ thick silicon wafer inclined $45^{\circ}$ to the incident beam ${ }^{2}$. The silicon wafer acts as a Fabry-Perot interferometer presenting only $\sim 4.5 \%$ loss along the signal path, while reflecting $\sim 50 \%$ of the LO power into the focal plane array. The instantaneous bandwidth of the diplexer is $\geq 1.3 \mathrm{GHz}$, an excellent match to the IF bandwidth 


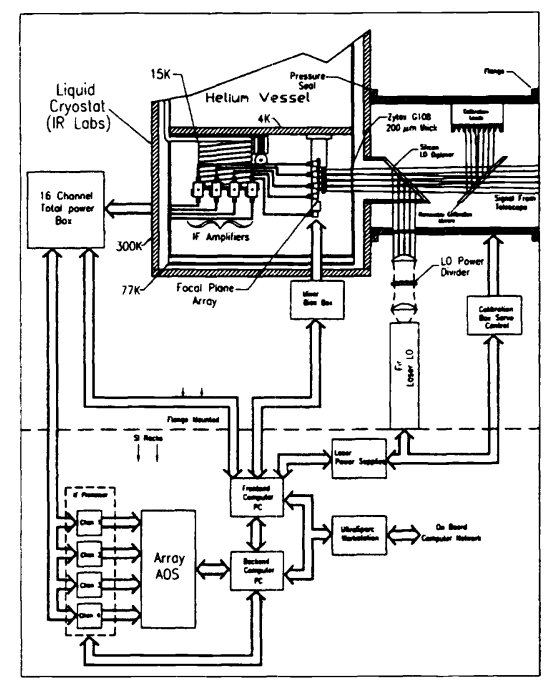

Figure 2. FAR Block Diagram

of the receiver. In order to reduce optical losses, the silicon diplexer will also serve as the vacuum window for the signal path.

An LO beam for each mixer will be produced by passing the FIR laser output through a quasi-optical phase grating. Optical phase gratings have been in use for over two decades. The application of phase gratings to the generation of multiple LO beams at $\mathrm{mm} /$ submm wavelengths is much more recent ${ }^{3,4}$. The beam to beam intensity variation can be $\leq 5 \%$ over a $13 \%$ bandwidth with a transmission efficiency of $83 \%^{4}$. Laser micromachining (see below) is ideally suited for the manufacture of these gratings and will be used to make gratings for FAR.

The combined signal and LO beams then enter a passively cooled cryostat. The cryostat will have a hold time greater than 24 hours with the system fully energized. With the receiver shutdown, the hold time will be $\geq 48$ hours. After passing through an infrared blocking filter, the beams illuminate the mixer subarray. The subarray is composed of 16 mixers stacked in a $4 \times 4$ matrix. Each mixer has a dielectric lens which lies in the telescope's focal plane. The lens diameter determines both the distance between mixers in the focal plane and the angular spacing of the beams on the sky. By cutting away flat sections from the sides of the lenses, the size of an array can be significantly reduced. We have conducted beam measurements on an $80 \mathrm{GHz}$ scale model of the horn/lens combination to determine the optimum amount of truncation. We propose to truncate the lenses at the $19 \mathrm{~dB}$ level $\left(\sim 3 \omega_{0}\right)$, which will set the beam spacing within each mixer subarray to $2.44 f \lambda$ and produce a negligible amount of loss $(<1.5 \%)$ and cross-talk between array pixels. The optical system has been optimized using commercial optical design software. Over the array aperture no significant off-axis aberrations are observed. The resulting subarray is quite compact $(31.2 \times 31.2 \mathrm{~mm})$. At the $1.9 \mathrm{THz}$ the diffraction limited beamsize is $\sim 16^{\prime \prime}$. The spacing between adjacent beams on the sky will be $\sim 32^{\prime \prime}$. At $3 \mathrm{THz}$ the array will have diffraction limited $\sim 10^{\prime \prime}$ beams with a projected beam spacing of $\sim 20^{\prime \prime}$.

The IF output of each mixer is amplified by a low-noise HEMT amplifier mounted on a $15 \mathrm{~K}$, He vapor-cooled, heat exchanger. (The IF frequency and bandwidth are set by the mixing device and the availability of laser LO lines.) After further amplification at room temperature, the IF output signals are fed into an IF processor which translates them to the array AOS center frequency. The receiver frontend and the four array AOS's are controlled by networked PC's. A PC running LINUX is used to process the 16000 channels of spectral data during flight and will serve as an interface between the instrument and the observatory's computer system.

\subsection{Mixing Devices}

NbTiN SIS junctions provide low-noise mixing at frequencies up to $\sim 1.2 \mathrm{THz}^{5}$. Above this frequency, their performance falls off quickly due to their relatively low gap frequency. Two alternative mixing devices for higher frequencies are hot electron bolometers (HEBs) and Schottky diodes. 


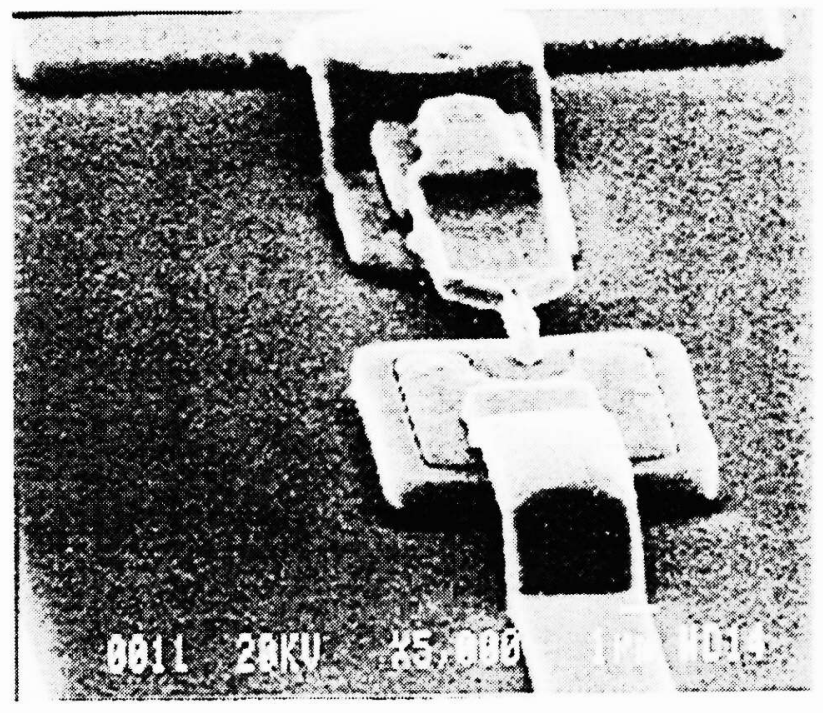

Figure 3. SEM micrograph of a $2.5 \mathrm{THz}$ MOMED device fabricated at JPL. (from Siegel et al. 2000

Corner cube mixers using whisker-contacted Schottky diodes have been widely used at THz frequencies for many years ${ }^{6,7}$. They have often been the only mixers available and have the advantage that they can be operated at room temperature. The main difficulties with them has been low-reliability, poor beam quality, and extremely tight assembly tolerances ${ }^{8}$. More recently, two groups have demonstrated waveguide mounted, "planar-whisker" contacted Schottky diode mixers at $2.5 \mathrm{THz}$, one group from Rutherford Appleton Laboratory and the other from the Jet Propulsion Laboratory. In particular, the monolithic membrane-diodes (MOMED) developed by JPL provide excellent performance $\left(T_{r e c} \sim 6,500 \mathrm{~K}\right.$ at $\left.2.5 \mathrm{THz}\right)$ and can be readily incorporated into focal plane arrays. A picture of a MOMED device is shown in Figure $3^{8}$.

Over the past few years, superconducting, niobium microbridge bolometers have been shown to produce low receiver noise temperatures at frequencies up to at least $2.5 \mathrm{THz}\left(T_{\text {rec }} \sim 1,850 \mathrm{~K} \text { at } 2.5 \mathrm{THz}\right)^{9,10,11}$. These devices make use of the large $d R / d T$ occurring at the resistive transition in a superconducting thin film. In such materials, the electrons are only loosely coupled to the crystal lattice and the electron-electron interaction is enhanced. When bombarded by photons these electrons can equilibrate at a temperature greater than the lattice. The electrons then can cool either through phonon coupling to the underlying lattice ${ }^{12}$ or through diffusion through normal metal contacts ${ }^{13}$. Which cooling mechanism dominates depends principally on the length of the bridge; diffusion cooling begins to dominate as the bridge length becomes smaller. Due to the heating of free electrons by the absorption of photons, these devices are referred to as Hot Electron Bolometers (HEB's). To date, the lowest noise temperatures at $\mathrm{THz}$ frequencies have been obtained using phonon cooled devices ${ }^{10}$, although diffusion cooled devices are not far behind ${ }^{14,15,11}$. The theoretical treatment by Prober (1993) suggests HEB devices will provide excellent performance up to several 10's of THz. Furthermore, these devices exhibit an essentially real impedance (of order 100 $\Omega$ ), making them much easier to match over large bandwidths to antenna and waveguide structures than their SIS counterparts.

The University of Virginia will design and fabricate the HEBs for the SOFIA arrays. The HEBs are fabricated on thin membranes and are placed in the center of the waveguide. Unlike the earlier work at JPL, which utilizes an ebeam lift-off process along with several self registered etching steps to fabricate the HEB elements, the HEBs will be fabricated utilizing a state-of-the-art focused ion beam system (FIB) to carve each $50-100 \mathrm{~nm}$ sized $\mathrm{Nb}$ microbridge in a single step. A typical $\mathrm{Nb}$ bolometer, fabricated with the FIB tool, is shown in Figure 4.

Compared to Schottky diode mixers, HEBs require several thousand times less LO power and have noise temperatures at least a factor of 2 less at $\sim 2.5 \mathrm{THz}$. However, HEBs must be cooled to at least $4 \mathrm{~K}$ and may be more susceptible to microphonics and LO amplitude variations than Schottky based mixers. We will make the FAR focal plane waveguide mixer mounts capable of supporting either MOMED or HEB devices. Since the output power provided by the DEOS laser system is between $50-100 \mathrm{~mW}$, there should be no problem in providing sufficient LO 


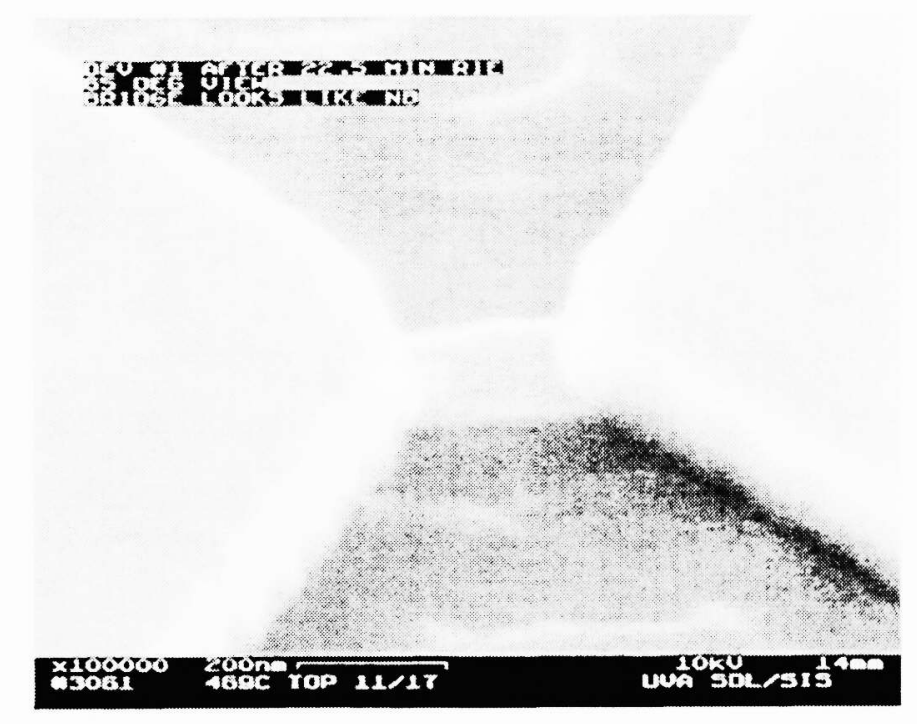

Figure 4. HEB fabricated with FIB process at UVa: The HEB is formed from a microbridge of niobium $10 \mathrm{~nm}$ thick, $200 \mathrm{~nm}$ long and $300 \mathrm{~nm}$ wide contacting gold pads $100 \mathrm{~nm}$ thick on either end. It is formed from a film of several layers deposited in a single vacuum using a focused-ion beam to cut away unwanted material. This microbridge exhibits a room-temperature resistance of $60 \Omega$ and has dimensions identical to those of other bridges fabricated the same way.

power for either type of device. The MOMED devices will be used to initially characterize the performance of the optics and waveguide mounts at room and cryogenic temperatures. HEBs will then be mounted in an identical FAR focal plane array and cooled to $4 \mathrm{~K}$. The performance of the HEB array, both in sensitivity and stability, will then be compared to what was achieved using MOMEDs. The arrays with the best overall performance will then be chosen for the flight instrument.

\subsection{Laser Micromachining of Submillimeter Waveguide Components}

\subsubsection{History}

Until recently, the fabrication of even single waveguide mixers at $\mathrm{THz}$ frequencies has been extremely difficult, if not impossible. However, the application of laser micromachining technology to this problem makes the fabrication of low-cost, high performance $\mathrm{THz}$ waveguide arrays tractable ${ }^{16}$. With this process, the desired waveguide structure is laser etched into silicon. The silicon structure can then be either metallized to make it behave like conventional air-filled waveguide or used as a mold for replicating a number of identical elements. The laser etching technique 17,18 operates independently of the crystal plane orientation and thereby permits a wide variety of structures to be made.

The University of Arizona and MIT Lincoln Laboratory established a Collaborative Research Agreement (CRDA) to investigate the application of laser micromachining to the fabrication of $\mathrm{THz}$ waveguide components. As a proofof-concept demonstration, we made a portion of an $810 \mathrm{GHz}$ and $2 \mathrm{THz}$ waveguide mixer block ${ }^{16}$. Figure 5 shows a portion of the $810 \mathrm{GHz}$ waveguide structure etched in 1 hour (a duplicate mating piece must be fabricated).

As a test of our fabrication and assembly process, and in an effort to test the proof-of-concept feedhorn for FAR, we performed beam pattern measurements on a laser micromachined $2 \mathrm{THz}$ corrugated feedhorn. Using the process described above, two, $2 \mathrm{THz}$ corrugated feedhorns were fabricated in an end-to-end arrangement as shown in Figure 6. The two horns are connected by a short $(0.164 \mathrm{~mm})$ section of circular waveguide. Beam pattern measurements were made on one horn using a calibrated black body source as the transmitter, while the other horn illuminated a $4.3 \mathrm{~K}$ silicon bolometer.

Figure 7 shows cross-scans on the prototype $2 \mathrm{THz}$ feedhorn. The plots indicate the feedhorn's beam is nearly Gaussian with no measurable sidelobes to the noise floor of the map $(\sim 11 \mathrm{~dB})$. There is a small asymmetry in 


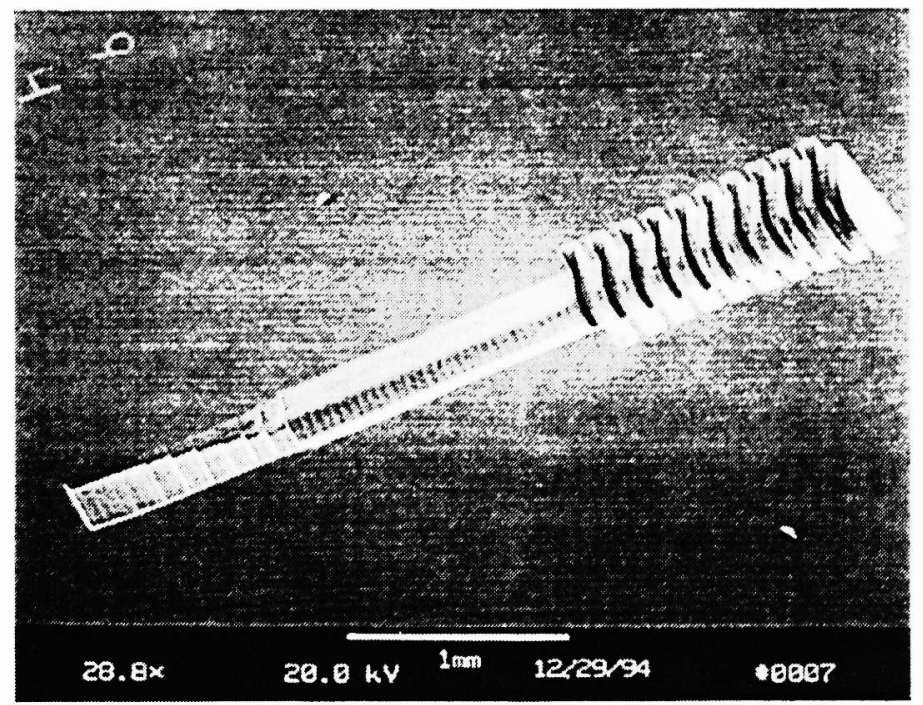

Figure 5. The structure was etched using 4.3 Watts of laser power focused into a $6 \mu \mathrm{m}$ spot in 200 Torr of chlorine gas. The laser beam was scanned at $5 \mathrm{~cm} / \mathrm{s}$ and incrementally moved 2 micrometers between line scans. Under these conditions, nominally $1 \mu \mathrm{m}$ shavings are removed per pass of the laser over the surface. The total etch time is one hour, not including the overhead time for pattern generation and stage motion.

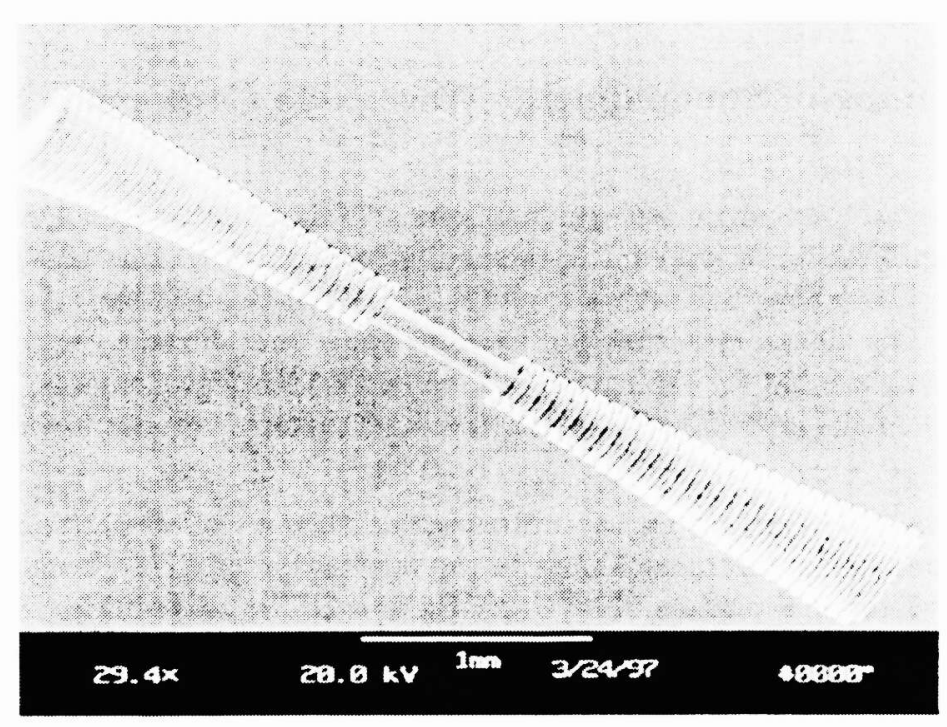

Figure 6. SEM micrograph of replicated version of $2 \mathrm{THz}$ waveguide structure. The original structure was etched using 3 Watts of laser power focused into a $4 \mu \mathrm{m}$ spot in 200 Torr of chlorine gas. The laser beam was scanned at $4 \mathrm{~cm} / \mathrm{s}$ and incrementally moved 2 micrometers between line scans removing $0.65 \mu \mathrm{m}$ shavings per pass of the laser over the surface. 


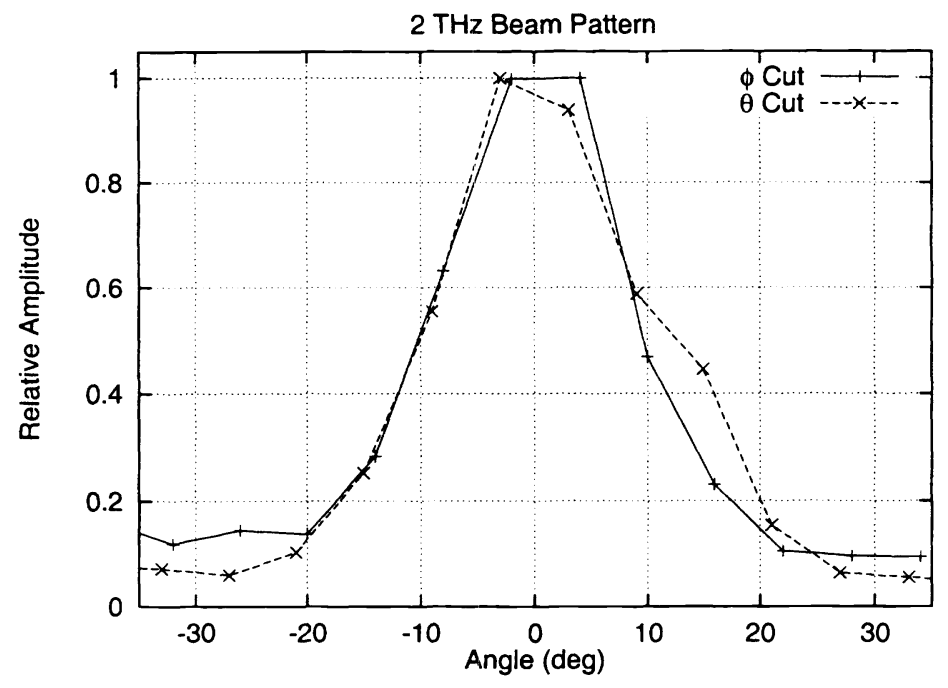

Figure 7. Beam cross scans of the micromachined $2 \mathrm{THz}$ feedhorn. The $\theta$ and $\phi$ cuts are orthogonal to each other.

the cross-scans, probably the result of a slight misalignment between the two halves of the horn block. (With the addition of interlocking features, the possibility of such misalignments will be eliminated.) The FWHM of the beam profile is $\sim 20^{\circ}$, close to the value of $19.3^{\circ}$ derived from the horn's beamwaist $\left(\omega_{\circ}=0.33 d\right.$ ), where $d$ is the diameter of the feedhorn aperture $(0.506 \mathrm{~mm})$ ). The differences between the theoretical and measured beam profiles are within the resolution limit and amplitude sensitivity of the test set-up.

Based upon the success of these efforts, the NSF has funded the construction of a laser micromachining system at Steward Observatory specifically designed for the fabrication of $\mathrm{THz}$ waveguide and quasi-optical components. This system will be used in the fabrication of the focal plane arrays and quasi-optical components for FAR.

\subsubsection{Focal Plane Array Concept}

The proposed array will utilize a $4 \times 4$ array of fixed-backshort, waveguide mixers. An assembly diagram of the mixer array is shown in Figure 8. The "Horn Block" consists of four, $1 \times 4$ corrugated feedhorn subarrays. Each subarray is formed by bonding two gold plated, silicon wafers in which mirror images of the corrugated feedhorns have been laser micromachined. Alignment between the two halves of a horn subarray is insured by posts and corresponding holes wet-etched in each half before laser machining.

Each horn is micromachined with a reduced-height waveguide transition (see Fig. 5). The reduced-height waveguide provides an excellent match to the real part of the MOMED or HEB impedance, permitting the use of only a single, fixed backshort. Electromagnetic finite element analysis software (HFSS) has been used in conjunction with $5 \mathrm{GHz}$ scaled models to determine the embedding impedance offered by the waveguide mount to a mixing device.

At high frequencies $(\geq 650 \mathrm{GHz}$ ) it becomes nearly impossible to manually mount detectors across small waveguide structures. An alternative approach is to fabricate the mixing elements on silicon nitride or GaAs membranes which subsequently are integrated with the horn structure. To date, this approach has been used successfully with SIS junctions up to frequencies of $850 \mathrm{GHz}{ }^{19}$ and Schottky diodes up to $2.5 \mathrm{THz}^{8}$. As in these successful design, the mixing devices are fabricated on membranes several times larger than the waveguide aperture.

Low pass filtering is provided by a suspended stripline circuit extending across the length of the membrane. Once onto the silicon substrate, coplanar waveguide is used to carry the IF signals to wire bonding pads on the edge of the Bolometer Array Block. Each IF output of the array is wire bonded to a microstrip matching network located just outside the periphery of the Bolometer Array Block (see Figure 11). Mixer bias is provided through the matching network. 


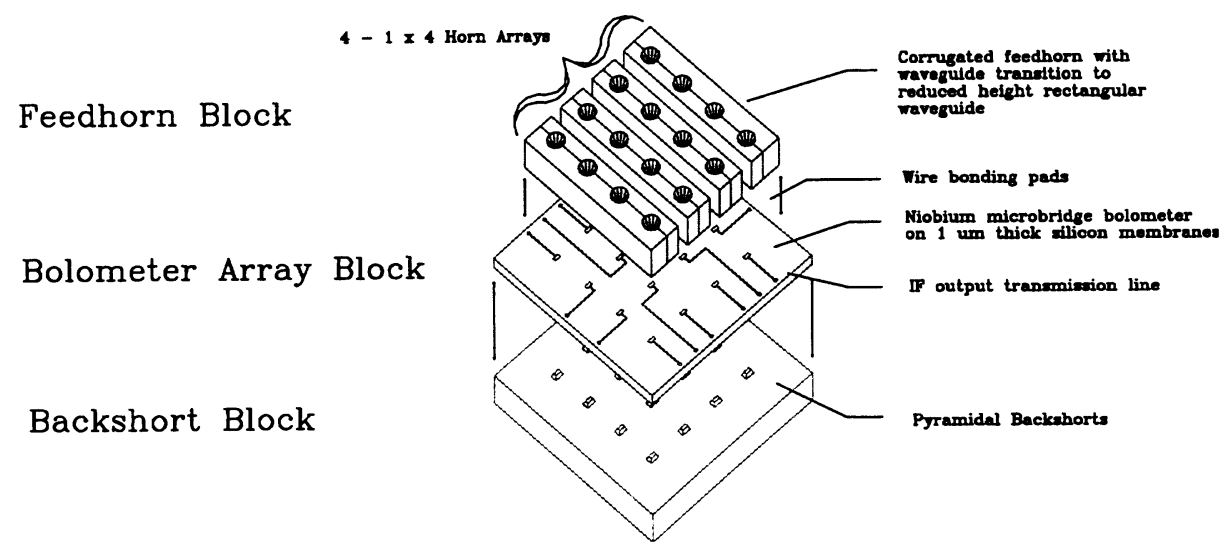

Figure 8. Assembly Drawing of the TeraHertz Array Mixer Block

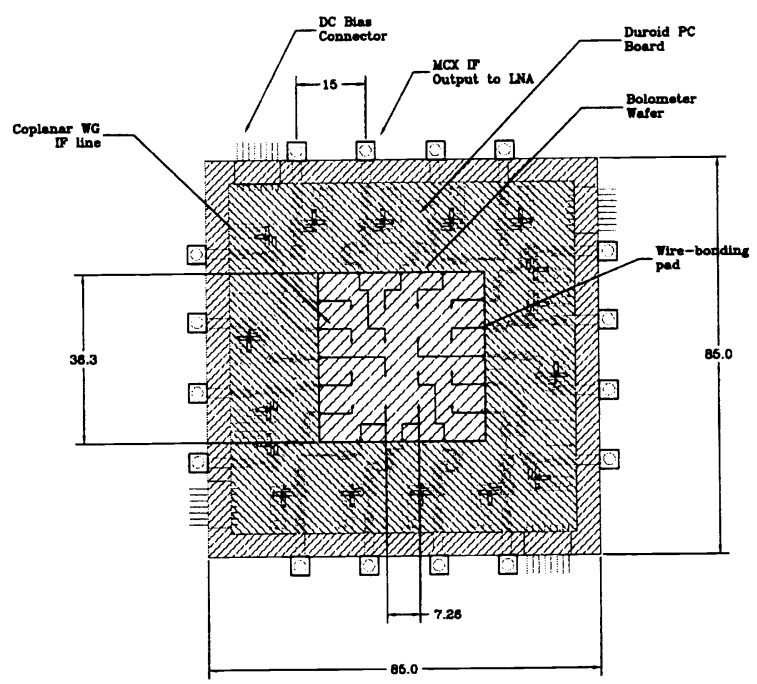

Figure 9. Bolometer Array Block showing with the Duroid PC Board containing the IF matching network and DC bias lines for the HEBs (or MOMEDs). 


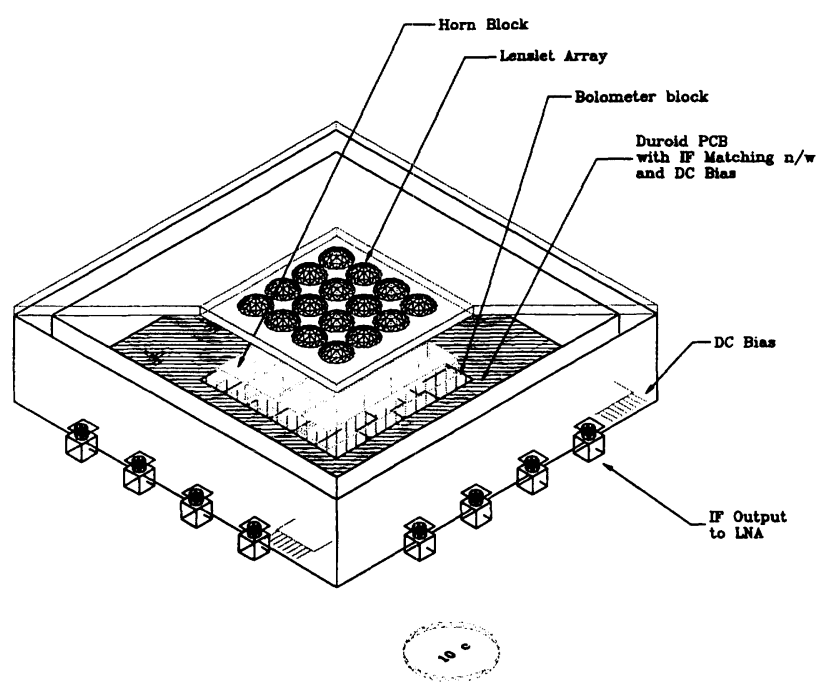

Figure 10. Overall 3-d isometric view of the mixer block with lenslet array, feedhorn section, junction and backshort blocks and the IF matching network PC board. The size of a dime is shown for comparison alongside the mixer block

The fixed rectangular backshort is laser micromachined on a pyramidal structure designed to fit the cavity behind the membrane. The pyramidal structures can be readily made by wet-etching silicon through an $\mathrm{SiO}$ mask evaporated on the wafer ${ }^{20}$. Once etched, the Backshort Wafer is gold plated.

We have optimized the design of the waveguide structures and IF circuitry using Hewlett Packard's High Frequency Structure Simulator (HFSS) and Microwave Design System (MDS). A CAD drawing of the complete $4 \times 4$ focal plane array is shown in Figure 10. The performance of the array will be optimized in the lab by trying different backshort lengths.

\subsection{Array AOS Backend}

In its final form, FAR will produce 16 independent channels of receiver output with an instantaneous bandwidth of $\geq 1 \mathrm{GHz}$. To create spectra from these channels, each must be processed by a backend spectrometer. A high resolution $\mathrm{THz}$ receiver requires a spectral resolution of $\sim 1 \mathrm{MHz}$. This translates to at least 1000 spectral points per array element. With a heterodyne array of 16 mixer elements, we gain efficiency by a factor of 16 , but now require $16 \times 1000$ channels to be taken simultaneously. Such a large number of channels is difficult to achieve with even the latest generation of autocorrelators and would require far more power and space than is available for an SI on SOFIA. Where wide bandwidths and high spectral resolution are required, Acousto-Optic Spectrometers (AOSs) are an attractive alternative technology.

AOSs have proven to be very capable backends for single-mixer receivers. The Submillimeter Wave Astronomy Satellite (SWAS) uses an AOS that provides $1400,1-\mathrm{MHz}$ channels in a very efficient, space-qualified package. AOSs from the University of Cologne are also successfully used at KOSMA on Gornergrat, at the IRAM 30-meter telescope on Pico Veleta, and at the Antarctic Submillimeter Telescope (AST/RO). The University of Cologne AOS group (led by Co-I Schieder) has extended the technology of the SWAS AOS and constructed a 4-channel, array-AOS (aAOS). Tests of the prototype aAOS have been conducted at the IRAM $30 \mathrm{~m}$ and CSO. The quality of the aAOS spectra are excellent and compare well to spectra taken with filterbanks and autocorrelators.

The University of Cologne is building four, 4-channel aAOS units of the type described above for use on SOFIA. The $4 \times 4$ AOS array will provide $16 \times 1 \mathrm{GHz}$ spectrometer channels for use with array receiver frontends. One such array will be the University of Cologne STAR, optimized for operation between 1 and $2 \mathrm{THz}$. The array described here (optimized for 2-3 THz) will also make use of this spectrometer system. 


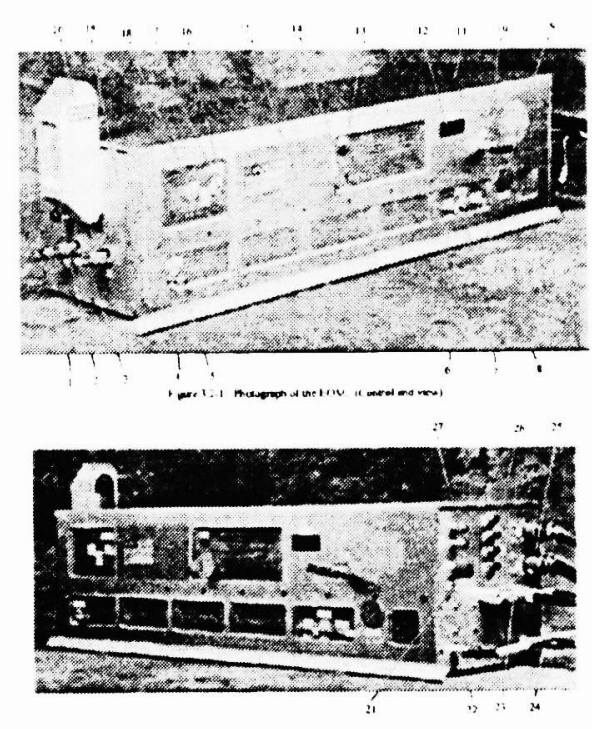

Figure 11. Photographs of GSFC/DEOS FIR Laser LO

\subsection{Laser Local Oscillator System}

The laser LO system for FAR will be supplied by NASA/GSFC as a result of a SBIR Phase II development awarded to DeMaria ElectroOptics Systems (DEOS), of Bloomfield, CT, for the design and construction of a waveguide $\mathrm{CO}_{2-}-$ pumped submillimeter local oscillator suitable for use on SOFIA. A photograph of the FIR laser system is presented in Figure 11.

This FIR laser is the first of its kind and utilizes the same ultra-long-life sealed-off pump laser technology which DEOS has implemented on a number of military flight programs, combined with an innovative absolute pumpfrequency locking technique and a compact folded FIR laser design. DEOS staff have demonstrated the requisite pump laser technology with sealed-off shelf lives in excess of 11 years and operating lives of $>25,000$ hours. Unlike conventional FIR lasers, this Stabilized Integrated Far-InfraRed (SIFIR) laser system will have an autonomous master controller and will operate completely sealed-off. The user of the SIFIR does not have to be knowledgeable about FIR lasers to operate the SIFIR. The design paradigms for the SIFIR are intended to reduce its operation to that of a scientific "appliance", as opposed to a finicky instrument. The pioneering concept for a SIFIR laser will lead to an order of magnitude reduction in volume $(\sim 6 " \mathrm{~W} \times 6 " \mathrm{H} \times 24 " \mathrm{~L})$ and weight $(\sim 35 \mathrm{lbs})$ over present technologies. These innovations are key to meeting the deployability requirements of ground-based/airborne THz radiometers. Further, DEOS will employ a thermally self-compensated design approach for the FIR laser. With this design approach, DEOS staff have demonstrated long-term frequency stability of $35 \mathrm{kHz}$ at $1.6 \mathrm{THz}$ over periods of days, and shortterm linewidths of $25 \mathrm{kHz}$ integrated over 3 minutes. With this laser system, FAR can achieve a velocity resolution of $\sim 4 \mathrm{~m} / \mathrm{s}$ ! Such high velocity resolutions may prove useful in studying such objects as planetary atmospheres and activity in proto-planetary disks. The high output power of the SIFIR laser will provide ample LO power to pump receiver arrays at $\mathrm{THz}$ frequencies.

\section{COMPLIANCE WITH S.I. ENVELOPE AND FAA REGULATIONS}

In Figure 12 FAR is shown mounted on the telescope flange. The instrument falls within the S.I. Envelope as defined in the CFR. The additional electronic equipment needed to run the instrument (aAOSs, IF processor, computers. and laser power supplies) fit within the two alotted S.I. racks. The total weight of the flange mounted instrument is $\sim 382 \mathrm{~kg}$, with a c.g. located $57.2 \mathrm{~cm}$ out, $16 \mathrm{~cm}$ up, and $8.6 \mathrm{~cm}$ to the right of where the IR beam emerges from the flange. The estimated power needed to run the instrument is $\sim 3.8 \mathrm{KVA}$, with most of the power being used to operate the FIR laser. The instrument cable drape will include $16 \mathrm{RG}-58$ coaxial cables, 4 power cables for the laser, 1 serial interface line, and a $115 \mathrm{~V} \mathrm{AC}$ power line. The cryostat will be a "wet" dewar designed to meet FAA safety requirements. 

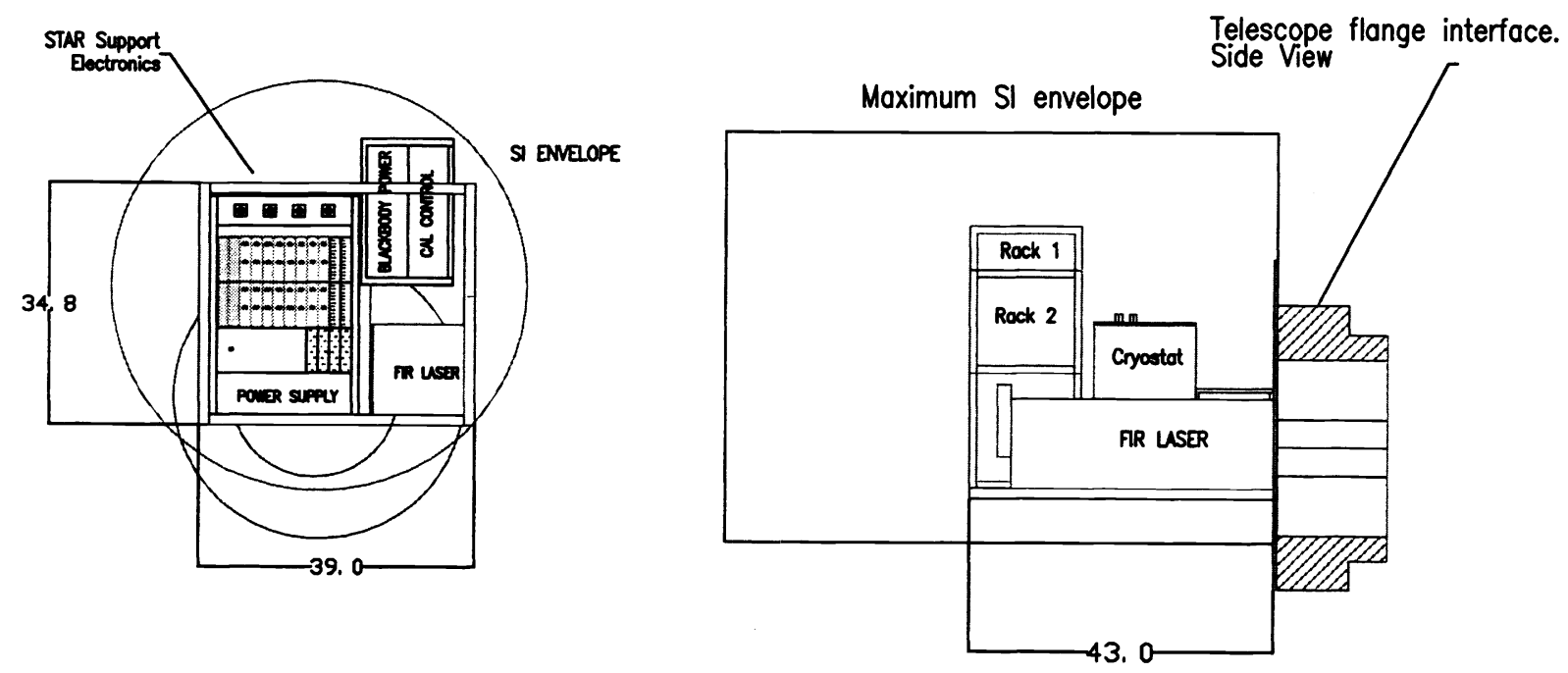

Figure 12. Compliance with the SI Envelope. All dimensions are in inches. (a) Back View of FAR bolted to the telescope flange. (b) Side View

\section{PLAN OF ACTION}

Our instrument development and operation plan has three phases. The goal of Phase 1 is to produce a fully functioning, $1 \times 4$ mixer subarray by June 2001. We will then propose FAR as a second generation instrument for SOFIA. Depending on the outcome of this proposal, the program enters Phase 2. In Phase 2 we stack 4 of the subarrays together to create the full $4 \times 4$ array. The full array with its support electronics and IF processing hardware will then be completed and characterized by October 2002. Phase 3 is the commissioning phase and includes initial flights on SOFIA.

\section{REFERENCES}

1. R. Borieko \& A. Betz, 1995, Astrophysical Journal, 454, 307.

2. E. Mueller \& J. Waldman, IEEE MTT, 1994, 42, 1891.

3. G. Delgada \& J. Johansson, 1995, ASP Conference Series, vol. 75.

4. T. Klein, G. Ediss, R. Güsten, H. Hauschildt \& C. Kasemann, 1997, Proc. 8th Int. Symp. on Space THz Technology, CofA, March 25-27, 1997.

5. J. Kooi, J. Stern, G. Chattopadhyay, H. LeDuc,B. Bumble \& J. Zmuidzinas, 1997, Proc. 8th Int. Symp. on Space THz Technology, C of A, March 25-27, 1997.

6. H. Krautle, E. Sauter \& G.V. Schultz, Infrared Physics, 1978, 18, 705.

7. A. Betz \& R. Borieko, 1995, Proc. 6th Int. Symp. on Space THz Technology, March 21-23, pp. 28-33.

8. P.H. Siegel, S. Martin \& S. Gaidis, IEEE Trans. MTT, 47, no. 5, 596.

9. R. Wyss, B. Karasik, W. McGrath, B. Bumble \& H. LeDuc, 1999, Proc. 10th Intern. Space THz Technol. Symp., University of Virginia, p. 214.

10. K. Yngvesson, E. Gerecht, C. Musante, Y. Zhuang et al., 1999, Proc. SPIE, Vol. 3795 (TeraHertz and GigaHertz Photonics), July.

11. B. Karasik, M. Gaidis, W. McGrath, B. Bumble \& H. LeDuc, 1997, Proc. 8th Int. Symp. on Space THz Technology, CofA, March 25-27.

12. E.M. Gershenshon et al., 1990, Sov. Phys. Superconductivity, 3(10), 1582.

13. D.E. Prober, 1993, Appl. Phys. Lett., 62, 2119.

14. A. Skalare et al., 1994, IEEE Trans. on Appli. Supercon., In Press. 
15. A. Skalare, W. McGrath, B. Bumble \& H. LeDuc, 1997, IEEE Transactions on Applied Superconductivity, in press.

16. C.K. Walker et al., 1997, Proc. 8th Int. Symp. on Space THz Technology, CofA, March 25-27, 1997.

17. T.M. Bloomstein \& D.J. Ehlrich, 1992, Appl. Physics Lett.,61, 706.

18. T.M. Bloomstein, 1996, Sc.D. Thesis, Massachusetts Institute of Technology.

19. J. Kooi, J. Pety, B. Bumble, H. LeDuc, P. Schaffer \& T.G. Phillips, 1998, IEEE MTT., 46, 151.

20. G. Rebeiz et al. 1987, Infrared and Milimeter Waves, 8, 1249. 